\title{
Relation von Norm und Praxis erziehungswissenschaftlicher Zugänge zu Inklusion und Partizipation in der Grundschule
}

\author{
Tanja Sturm (D)
}

Eingegangen: 29. Oktober 2020 / Angenommen: 28. Oktober 2021 / Online publiziert: 7. Januar 2022 (C) Der/die Autor(en) 2022

Zusammenfassung Der Beitrag gibt einen Einblick in die Konzeption der Relation von Normen und Praxis von Inklusion und Partizipation, die im aktuellen erziehungswissenschaftlichen Diskurs der Grundschulpädagogik in unterschiedlichen aufgerufenen Zugängen beschrieben werden. Exemplarisch werden die Inklusions- und Partizipationsverständnisse der evidenzbasierten Bildungsforschung, der auf Foucault aufbauenden Dis_Ability Studies und der Praxeologischen Wissenssoziologie vergleichend vorgestellt. Es wird aufgezeigt, dass in den Ansätzen nicht nur die Genese von Normen und Praxen bei je unterschiedlichen sozialen Akteur:innen verortet wird, sondern auch deren (sozialwissenschaftliche) Bewertung. Dies geht seinerseits im Forschungsprozess mit unterschiedlichen Partizipationsmöglichkeiten derjenigen, deren Praxis Gegenstand der Forschung ist, einher.

Schlüsselwörter Norm · Praxis · Inklusion · Partizipation ·

(Sozialwissenschaftliche) Bewertung

Tanja Sturm $(\bowtie)$

Philosophische Fakultät III, Martin-Luther-Universität Halle-Wittenberg, Halle (Saale), Deutschland

E-Mail: tanja.sturm@paedagogik.uni-halle.de 


\title{
Relation of norm and practice of educational approaches to inclusion and participation in elementary school
}

\begin{abstract}
The article provides insight into the conceptualization of the relation of norms and practice of inclusion and participation in different approaches used in current education research of primary education. As examples, the understandings of inclusion and participation of evidence-based educational research, Dis_Ability Studies based on Foucault, and the Praxeological Sociology of Knowledge are presented comparatively. It is shown that in these approaches not only the genesis of norms and practices is located in different social actors, but also their (sociological) evaluation. This goes hand in hand with different possibilities of participation for those whose practice is being researched.
\end{abstract}

Keywords Norm · Practice $\cdot$ Inclusive education · Participation · (Social science) evaluation

Die Frage nach der Relation von Normen und Praxen stellt seit der Konstituierung der Erziehungswissenschaft als universitärer Disziplin ein wiederkehrendes bzw. vielfach bearbeitetes Themen- und Gegenstandsfeld dar (vgl. in jüngeren Arbeiten z.B. Koller 2017; Meseth et al. 2019). Zunächst wurde dies entlang der Begriffe Erziehung und Bildung von Immanuel Kant und Wilhelm von Humboldt diskutiert. Deren philosophisch begründeten Ideale, Normen und Erwartungen fokussieren v. a. die Frage, wie die Gesellschaft durch Bildung und Erziehung der einzelnen zum Besseren verändert werden kann und soll. Dass dies in der praktischen Umsetzung nicht gelingt, führt Émile Durkheim, der Kants Erziehungsbegriff u. a. als normativ und kulturell - statt universalistisch - geprägt kritisierte, darauf zurück, dass lediglich die Lebensbedingungen resp. Lernvoraussetzungen ausgewählter Personengruppe berücksichtigt würden (vgl. Koller 2017, S. 117 ff.). Wenngleich Durkheims soziologische Kritik an diesen frühen philosophischen Erziehungs- und Bildungsverständnissen impliziert, dass - so die Lebensbedingungen aller berücksichtigt würden - die Ideale pädagogisch bzw. sozial erreicht werden können, muss auch heute konstatiert werden, dass Normen und Praxen mehrheitlich als diskrepant beschrieben werden. Erklärungsangebote für diese Differenzen finden sich in sozialwissenschaftlichen Metatheorien, vor deren Hintergrund die aktuellen erziehungswissenschaftlichen Diskurse und die von ihnen aufgerufenen Gegenstandsverständnisse, wie Inklusion und Partizipation, definiert werden. In dem Diskurs zu Inklusion - und mit ihm verbunden dem zu Partizipation - wird die Frage nach dem Verhältnis von Normen und Praxen derzeit zugespitzt diskutiert (vgl. Fritzsche et al. 2021). Die fachwissenschaftliche - ebenso wie die gesellschaftliche Auseinandersetzung mit Inklusion erfolgt fast durchgängig unter Verweis auf die Konvention über die Rechte von Menschen mit Behinderungen, kurz UN-BRK, der Vereinten Nationen (2008 [2006]), die als normativer Bezugspunkt fungiert, wenngleich nicht immer eindeutig abgegrenzt wird, ob in moralischer, rechtlicher und/ oder politischer Hinsicht (vgl. Weyers 2019). Mit diesem menschenrechtlichen Bezug wird eine übergeordnete und zugleich universalistische Perspektive aufgemacht, 
zu der sich auch wissenschaftliche Praxen und ihre Normen in Beziehung setzen müssen, so sie für sich nicht den Anspruch erheben, losgelöst von diesen zu agieren (vgl. Wrana 2019).

Im Anschluss an die bildungspolitisch initiierten Reformen zur Gestaltung inklusiver Schulsysteme, die im Zuge der Ratifizierung der UN-BRK durch Bund und Länder erfolgte, verbreitert und differenziert sich auch der erziehungswissenschaftliche Fachdiskurs zu Inklusion und Partizipation. Vor allem Inklusion wird aus unterschiedlichen grundlagentheoretischen Perspektiven, wie u.a. der Empirischen resp. evidenzbasierten Bildungsforschung (vgl. z. B. Grosche 2015), der Kritischen Erziehungswissenschaft (vgl. z. B. Katzenbach 2015), dem interdisziplinären Forschungsstrang der Dis_Ability Studies (vgl. z. B. Köbsell 2015; Waldschmidt 2012), anerkennungstheoretischen Ansätzen (vgl. z. B. Fritzsche 2018) und der Praxeologischen Wissenssoziologie (vgl. z. B. Sturm 2015) bearbeitet. Diesen unterschiedlichen Zugängen, die - vergleichbar anderen in der Grundschulpädagogik bearbeiteten Gegenständen - in unterschiedlichen sozialwissenschaftlichen bzw. Metatheorien verankert sind, zeichnen sich durch die Gemeinsamkeit aus, dass Inklusion als Überwindung von Exklusion resp. Marginalisierungen sowie behinderter akademischer und sozialer Partizipation verstanden wird (vgl. Ainscow 2008). Die theoretischen und empirischen Auseinandersetzungen der Relation von Norm und Praxis von Inklusion und Partizipation unterscheiden sich hingegen und erfolgen entlang je eigener begrifflicher Kategorien, die in diesem Beitrag exemplarisch für drei Ansätze vergleichend dargelegt werden: für die evidenzbasierte Empirische Bildungsforschung, die in einem rational-technologischen Verständnis fundiert ist (Abschn. 1), für die an Foucault anknüpfende macht- und subjektivierungstheoretische Diskurslinie der Dis_Ability Studies (Abschn. 2) und für den kultursoziologischen Ansatz der Praxeologischen Wissenssoziologie (Abschn. 3). Abschließend werden die drei Ansätze, die sich wesentlich darin unterscheiden, welche sozialen Akteur:innen als Normen und Praxen von Inklusion und Partizipation hervorbringend sowie diese bewertend gesehen werden, miteinander verglichen. Dies erfolgt entlang der Frage, inwiefern Inklusion und Partizipation nicht nur als Gegenstände sondern auch als Prinzipien von Forschungsprozessen verhandelt werden, um diese abschließend in ihrer Bedeutung für die Grundschulpädagogik zu diskutieren (Abschn. 4).

\section{Evidenzbasierte Bildungsforschung - statistisch generierte ,Inklusions- und Partizipations-Normen' für die Praxis}

Die evidenzbasierte Bildungsforschung steht für ein Forschungsparadigma, das in der Schul- und Unterrichtsforschung in den vergangenen 20 Jahren stark an Bedeutung gewonnen hat; dies gilt entsprechend für die grundschul- und unterrichtsbezogene Inklusions- und Partizipationsforschung (vgl. z. B. Müller und Pfrang 2021). Bezogen auf die letztgenannte ist es v. a. das Programm resp. Modell des Responseto-intervention, kurz RTI, das für diese Schulstufe erarbeitet wurde und in den USA und in Deutschland vielfach angewandt wird (vgl. z. B. Huber und Grosche 2012; Vossen und Krizan 2021). RTI wird als „konzeptuelles Gerüst“ verstanden, das sich aus „Einzelkomponenten“ (Voß et al. 2014, S. 115) zusammensetzt und an 
den Zielen der Prävention und der Integration von Schüler:innen, denen sonderpädagogischer Förderbedarf attestiert wird, orientiert ist. Der evidenzbasierte Ansatz ist in den Grundannahmen der Empirischen Bildungsforschung (vgl. z. B. Gräsel 2011) verankert und versteht sich als ,alternative Form der Feststellung von Lernund Entwicklungsbeeinträchtigungen“ (Voß et al. 2014, S. 115). Das sich darin andeutende Verständnis von Inklusion lässt sich, folgt man Michael Grosche (2015), einem der zentralen Vertreter:innen von RTI im deutschsprachigen Raum, als „Gesamtgefüge" verstehen, das empirisch zu fassen, er auch mehrebenenanalytisch als für nicht möglich erachtet. Er schlägt vor, stattdessen einzelne Elemente von Inklusion wissenschaftlich zu betrachten. Diese Elemente leitet er aus Definitionen aus (Fach-)Texten ab und ordnet diese den drei Begriffen Kulturen, Strukturen und Praktiken des deutschsprachigen ,Index für Inklusion“ (Hinz und Boban 2003) zu. Das RTI-Modell versteht er als ,,inklusive Struktur“ (Grosche 2015, S. 34), die eine „steuernde Funktion“ für die Gestaltung von Inklusion innehat.

Die Empirische Bildungsforschung, auf die die evidenzbasierte Bildungspolitik und -praxis - einige, wie die Vertreter:innen des RTI-Modells sprechen auch von evidenzbasierter Bildungsforschung - aufbauen, versteht sich als ,interdisziplinäres Forschungsfeld“" (Gräsel 2011, S. 25), dessen zentraler Gegenstand institutionalisierte Formen der Bildung, verstanden als prozesshafter Kompetenzerwerb, sind. Das Arbeitsfeld, an dem u.a. Psychologie, Soziologie, Ökonomie, Erziehungswissenschaft und Fachdidaktiken beteiligt sind, zeichnet sich durch erkenntnistheoretische Zugänge aus, die rational-technologisch fundiert sind und v. a. mit standardisierten Methoden arbeiten (vgl. Tillmann 2016). Das methodische Vorgehen des Ansatzes wurde einem, in den 1960er-Jahren für die Medizin und Pharmazie entwickelten entlehnt, in dem die Effektivität der Wirksamkeit von Medikamenten durch Kontrollgruppenvergleiche gemessen wird. Die Wirksamkeit einer (pädagogischen) Maßnahme gilt entsprechend als erfolgreich bzw. ist evident, wenn sie aufseiten der Schüler:innen mehrheitlich das gewünschte Ziel hervorbringt, z. B. ein spezifisches Verhalten. Der Zugang, der auf einem positivistischen und rational-technologischen Verständnis von Sozialität aufbaut, stellt ,auf der Grundlage von large-scale Untersuchungen statistisch fest [...], dass [eine Maßnahme] wirkt“" (Tröhler 2019, S. 14; Anm. TS), nicht aber, warum sie (nicht) wirkt. Die auf diese Weise legitimierten Unterrichts- oder Fördermethoden können innerhalb des Ansatzes insofern als Norm bezeichnet werden, als sie in die Praxis zu überführen sind, also Praxis werden sollen. Das Verhältnis von Norm und Praxis ist entsprechend hierarchisch und linear konzipiert, in dem die mittels statistischer Prüfung ermittelten Normen eine Anleitung für die Praxis darstellen. Damit wird nicht nur angenommen, dass das „Technologiedefizit der Erziehung“ (Luhmann und Schorr 1982) und das Aushalten von Ungewissheit und Kontingenz in pädagogischen Interaktionen und Praxen überwunden werden kann, sondern auch, dass die je spezifischen Kontextualisierungen von Lehr-Lernsettings und die Erfahrungen der sozialen Akteur:innen ausgeblendet werden.

Das RTI-Modell ist insofern differenzierter als die dargelegte Figur statistisch abgesicherter Wirksamkeit unterrichtlicher Methoden, als es ein mehrstufiges, durchgängig evidenzbasiertes Vorgehen beschreibt, das unterschiedliche Diagnostik- und Fördermaßnahmen umfasst, wenn die Folge (response) von Lehrprozessen (inter- 
vention) nicht wie statistisch naheliegend, lernen ist. Das Modell unterscheidet drei Ebenen: die des regulären Unterrichts, die der fokussierten und die der speziellen Interventionen. Sie unterscheiden sich entlang der Häufigkeit des Einsatzes (in)formeller diagnostischer Screenings, die mittels norm- und kriteriumsorientierter Tests durchgeführt werden, sowie der Förderintensität, die zusätzlich zum grundständigen Unterrichtsangebot (Ebene 1) erfolgt. Das Modell ist an dem Ziel ausgerichtet, frühzeitig zu erkennen, dass den Schüler:innen, die die schulischen Leistungs- und Verhaltenserwartungen nicht erfüllen, zusätzliche Lehrangebote unterbreitet werden (vgl. Huber und Grosche 2012). Hier wird ein Verständnis von Inklusion erkennbar, das darin besteht bzw. daran ausgerichtet ist, dass alle Schüler:innen die schulischen Bildungserwartungen erfüllen. Für Schüler:innengruppen, denen dies innerhalb des allgemeinen Unterrichtsangebots nicht gelingt, werden spezifische kompensatorische Maßnahmen entwickelt und erprobt.

Die im Rahmen des Rügener Inklusionsprojekts, das im RTI-Modell fundiert ist, erkannten Diskrepanzen zwischen ,theoretischer Vorgabe und praktischer Umsetzung“ (Voß et al. 2014, S. 128) erklären die Autor:innen u.a. damit, dass es den „Lehrkräften noch nicht [gelingt], die Potenziale der angewendeten Konzepte, Materialien und Messverfahren voll zu nutzen“. Die im Vergleich größere Diskrepanz zwischen Theorie bzw. den statistisch ermittelten Normen und der Praxis, die sich in der Rügener Studie gegenüber vergleichbaren Untersuchungen in den USA zeigen, führen sie u. a. darauf zurück, dass mit dem RTI-Modell weitere Veränderungen einhergingen, die in den USA bereits etabliert sind, wie u. a. den Rahmenbedingungen, die ,räumliche, sächliche und personelle Fragen betreffen“ in Deutschland jedoch noch nicht. Diese, z.T. auch als ,systemische ,Beharrungstendenzen““ (Thiel et al. 2014, S. 134; Herv. im Orig.) der schulischen Akteur:innen erklärte Differenz wäre zu beforschen, so die Vertreter:innen dieses Ansatzes.

Zusammenfassend ist festzuhalten, dass die evidenzbasierte Bildungsforschung eine hierarchische und lineare Relation von Norm und Praxis von Inklusion und Partizipation aufmacht. Die statistisch legitimierten, von der Wissenschaft erarbeiteten und überprüften Normen fungieren dabei als Maßstab zur Bewertung der Praxis; Bewertungen der Praxis durch diejenigen, die diese hervorbringen bzw. gestalten, werden nicht als gleichwertig betrachtet (vgl. Wittich und Kuhl 2021). Inklusion und Partizipation - bzw. Einzelelemente - werden auf der Grundlage theoretischer Arbeiten operationalisiert und Maßnahmen zu ihrer Erreichung entwickelt und überprüft. Neben diesen, hier zugespitzt formulierten Perspektiven finden sich in der Empirischen Bildungsforschung auch relativierende Positionen, die die soziale Situierung von Bildung und Erziehung und/oder die Begrenztheit der wissenschaftlichen Ergebnisse für die Umsetzung in der Praxis reflektieren (vgl. Hartmann et al. 2016).

\section{Macht- und subjektvierungstheoretische Perspektiven der Dis_Ability Studies auf Normen der Inklusion und Partizipation in schulisch- unterrichtlichen Praxen}

Auch die Dis_Ability Studies verstehen sich als transdisziplinären Forschungsstrang, an dem u.a. die Soziologie, die Rechtswissenschaften und die Erziehungswissen- 
schaft beteiligt sind und der sich aus der Behindertenbewegung der 1970er und 1980er-Jahren zunächst im englischsprachigen und seit der Jahrtausendwende auch im deutschsprachigen Raum (vgl. Tervooren 2000) entwickelt hat. Er zeichnet sich durch einen gesellschaftskritischen und emanzipativen Anspruch aus (vgl. Köbsell 2015; Waldschmidt 2005). Der Diskursstrang, der Erziehung und Bildung fokussiert, die Dis_Ability Studies in Education, hat in den letzten Jahren in der deutschsprachigen Inklusionsforschung an Bedeutung gewonnen (vgl. z. B. Buchner et al. 2015; Hirschberg und Köbsell 2021). Vergleichbar dem englischsprachigen Diskurs (vgl. z.B. Tremain 2008) wird dabei auf die gesellschaftskritischen, macht- und subjektivierungstheoretischen Arbeiten Michel Foucaults (1976) zurückgegriffen (vgl. für eine Übersicht: Waldschmidt 2020), entlang derer Normen und Normativität sowie die ihnen inhärenten Ausschlüsse, Marginalisierungen resp. Behinderungen in Diskursen und Praxen beschrieben und Perspektiven ihrer Überwindung aufgeworfen werden.

Die Dis_Ability Studies, die sich wesentlich aus der Kritik an einem essentialisierenden Verständnis von Behinderung - das Behinderung als individuelles (körperliches und/oder psychisches) Merkmal, das v. a. mit Hilfe von Expert:innen und unter Aberkennung von Autonomie in Sondereinrichtungen gesellschaftlich bearbeitet wird - entwickelt haben, analysieren v. a. ,gesellschaftliche Normierungs-, Disziplinierungs- und Subjektivationspraktiken“. Letztgenannte werden dabei in ihrer historischen und kulturellen „Relativität und Kontingenz von Behinderung“ (Waldschmidt 2007, S. 55) resp. Abweichungen beschrieben. Vergleichbar zu rassismuskritischen Forschungsansätzen stehen die Mehrheitsgesellschaft und ihre Hervorbringung von ,Anderssein“ im Fokus des Ansatzes (vgl. z. B. Linnemann et al. 2013). Neben expliziten werden v. a. den Diskursen und Praxen inhärente Normen bzw. Normalitätserwartungen menschlicher Fähigkeiten (ableness) betrachtet, mit dem Ziel, die ihnen inhärenten Privilegierungen und Behinderungen von Inklusion und Partizipation zu rekonstruieren (vgl. Waldschmidt 2005). Praxen werden - im Anschluss an Foucault (2020) und seiner erziehungswissenschaftlichen Rezeption - als Diskurse bzw. als diskursiv hervorgebracht verstanden. Entlang der ihnen inhärenten Normen werden (hierarchische) Unterscheidungen von Subjekten, in Schule und Unterricht v. a. der Schüler:innen, vorgenommen. Die Rekonstruktion und Thematisierung dieser kulturellen Subjektivierungs-, Ungleichheits- und Machtverhältnisse sowie ihre alltagspraktischen Folgen für die Subjekte, stellen das Kernanliegen des Forschungszugangs dar. Der gesellschaftskritische und emanzipative Ansatz vergleicht diese Rekonstruktionen mit einem Inklusions- und Partizipationsverständnis, das die Überwindung von Aussonderung in spezielle Institutionen (z. B. Sonderschulen), die rechtliche Gleichstellung sowie die Etablierung einer Selbstverständlichkeit gemeinsamer Erziehung und Bildung von Kindern und Jugendlichen mit und ohne Behinderungen (vgl. Köbsell 2012) als Normen aufruft. Letztgenannte sind mit den Zielen von Inklusion und Partizipation, wie sie in der UN-BRK formuliert werden, weitgehend identisch. Die Dis_Ability Studies in Education stellen mithin ein Forschungsprogramm dar, das die unterschiedlichen schulisch-unterrichtlichen Diskurse und Praktiken zu Inklusion und Partizipation, zu denen u. a. Gesetzestexte, Fachdiskurse, Schulprogramme und Praktiken zählen, auf die ihnen inhärenten Normen, 
Macht- und Subjektivierungsverständnisse untersucht und diese mit Menschen- und Bürger:innenrechten sowie machttheoretischen Ausführungen vergleicht.

Analysen der Dis_Ability Studies in Education umfassen u. a. solche (Verdopplung von Analyse) kultureller Konstruktionen von Normalität und Abweichung in schulischen Bildungs- und Rahmenplänen sowie Curricula. Für US-Amerikanische Curricula konnte das ihnen inhärente Idealbild eines Schülers rekonstruiert werden, das an sogenannten höheren Bildungsidealen orientiert ist und sich als ,ego and super-ego of European-American male“ (Erevelles 2005, S. 433) darstellt. Die aufgerufenen Leistungsverständnisse und normativen Erwartungen der Leistungsfähigkeit stellen dabei zentrale Formen der (Selbst-)Disziplin in Schule und Unterricht dar - auch über sogenannte inklusive Kontexte hinaus (vgl. Ricken 2018). Für den bildungspolitischen Diskurs zu schulischer Inklusion konnte Uwe Becker (2015) Formen der erwarteten Anpassung von Schüler:innen mit Behinderungen an eine regelschulische Normalität aufzeigen. Mit anderen Worten, das programmatische, von der Bildungspolitik aufgerufene Verständnis von Inklusion bringt in der Praxis Exklusions- und/oder Marginalisierungsformen hervor (vgl. z. B. Wansing 2007). Hieran anschließend lässt sich mit diesem Ansatz eher fragen, ob und inwiefern die UN-BRK und die durch sie angestoßenen gesellschaftlichen Diskurse einen Beitrag zu einer Verschiebung der Relation von Normalität und Abweichung in Schule und Unterricht, also der Praxis leistet bzw. leisten kann, und weniger, ob Exklusion umfänglich überwunden ist.

Im Vergleich zum wissenschaftlichen Zugang der evidenzbasierten Forschung werden Normen im Ansatz der Dis_Ability Studies nicht statistisch begründet, sondern einerseits als diskursiv hervorgebrachte Unterscheidungen von Normalität und Abweichung und andererseits im Ideal egalitärer Differenz und Zugehörigkeit resp. gesellschaftlich-kultureller Partizipationsmöglichkeiten jenseits von Ungleichheitsunterscheidungen verstanden. Erstgenannte verweisen darauf, dass sich die Praxis selbst durch Normen auszeichnet und diese kontinuierlich und diskursiv hervorbringt, während letztgenannte Norm für einen Bewertungsmaßstab steht, der, den gesellschaftskritischen Analysen Foucaults folgend, mit den Bürger:innen- und Menschenrechten resp. dem emanzipativen Anspruch der Überwindung gegebener Macht- und Subjektvierungsverhältnisse formuliert wird. Letztgenanntes dient dabei als Vergleichsmaßstab; die Wertung entlang deskriptiv-analytischer Darstellungen der aufgerufenen Normen und ihrer subjektivierenden Folgen kann durch Wissenschaftler:innen und Praktiker:innen gleichermaßen erfolgen (vgl. Hirschberg und Köbsell 2021). Sie orientiert sich v. a. an dem Ziel, inhärente Normen beschreibund so reflexiv und mit emanzipativem Anspruch zugänglich zu machen. Weder Diskurse noch Praxen werden dabei als individuell zu verändern konzipiert, vielmehr werden sie als gesellschaftliche verstanden, die v. a. politisch zu verändernd sind. 


\section{Praxeologische Wissenssoziologie - Bearbeitung notorischer Diskrepanz von Norm und Praxis von schulisch-unterrichtlicher Inklusion und Partizipation}

Der kultursoziologische Ansatz der Praxeologischen Wissenssoziologie wurde in den letzten Jahren von Ralf Bohnsack $(2017,2020)$ auf der Grundlage empirischer und theoretischer Arbeiten zur Rekonstruktion handlungsleitender Orientierungen von Milieus, die in Organisationen, wie der Schule, hervorgebracht werden, differenziert. Die grundlegende ,notorische Diskrepanz von Norm und Habitus“ (Bohnsack 2017, S. 103) resp. von Norm und Praxis, die die von Karl Mannheim (1980) beschriebene Leitdifferenz der Wissenssoziologie aufgreift, übertragt Bohnsack (2017, S. $102 \mathrm{ff}$.) auf die propositionale und performative Logik. Einer proponierten Logik folgen i.d.R. sprachlich explizierbares und damit reflexiv zugängliches Wissen, zu dem Normen, Erwartungen und Ideale zählen sowie Common-Sense-Theorien, die eine Verständigung im Alltag ermöglichen. Konjunktives oder habituelles Wissen, der Modus Operandi der Handlungspraxis, folgt hingegen einer performativen Logik und wird als „Orientierungsrahmen im engeren Sinne“ (Bohnsack 2017, S. 103; Herv im Orig.) bezeichnet, d.h., obwohl die sozialen Akteur:innen über dieses, meist vorbegriffliche Wissen verfügen, können sie es i.d.R. nicht explizieren, z.B. wie sie sich körperlich bewegen und dabei von anderen als Frau/Mann/Divers erkannt werden. Beide Logiken, die der Norm und die des Habitus, stehen in einem „Spannungsverhältnis“ (Bohnsack 2017, S. 103) zueinander. Diese „,notorische Diskrepanz" (ebd.) wird in der Alltagspraxis von den sozialen Akteur:innen explizit, aber v. a. implizit, also handlungspraktisch, bearbeitet. Die habitualisierte Bearbeitung des Spannungsverhältnisses von Norm und Habitus beschreibt Bohnsack (2017, S. 103; Herv. im Orig.) als „Orientierungsrahmen im weiteren Sinne“.

Organisationsmilieus, also Milieus, die in Organisationen generiert werden, zeichnen sich im Unterschied zu außerorganisationalen Milieus durch eine konstitutive Rahmung aus. Mit dieser beschreibt Bohnsack (2017, S. 136) die gesellschaftlichen Bedeutungen und Aufgaben der Institution bzw. Organisation, die in kodifizierter und damit expliziter Form vorliegen. Für die Schule finden sich diese u. a. in Schulgesetzen und Bildungs- und Rahmenplänen, die die Erwartungen an die sozialen Rollen der Akteur:innen formulieren. Diese stellen Bezugspunkte für institutionelle Entscheidungen und auch Unterscheidungen wie z.B. Schüler:innen entlang von Leistungen und Verhalten dar. Dabei ist dieser konstitutive Rahmen pädagogischer Organisationen weder kausal noch deterministisch, vielmehr sind die Akteur:innen gefordert, sich explizit und/oder implizit in ihrer Praxis und vor dem Hintergrund ihrer (beruflichen) Erfahrungen mit diesen Normen und Erwartungen auseinander zu setzen und - wenn möglich - organisationale konjunktive Erfahrungsräume, also Routinen mit den Schüler:innen zu etablieren. Erst die rekonstruktiven Analysen der jeweiligen Praxis - des konjunktiven Orientierungsrahmens im weiteren Sinne zeigen, wie die komplexe Mehrdimensionalität sowie die Diskrepanzen von Normen und Habitus von den Akteur:innen bearbeitet werden und ob es gelingt, Milieus zu generieren, die den sozialen Akteur:innen, die sich entlang ihrer sozialen Rollen begegnen, Verlässlichkeit im Miteinander ermöglichen. 
Inklusion und Partizipation lassen sich mit den Kategorien der Praxeologischen Wissenssoziologie für beide, für Normen und Praxen, erkennen und beschreiben, also für in expliziter und kodifizierter Form vorliegende, die durch die Institution, in der die Praxis hervorgebracht wird, formuliert werden und/oder von den sozialen Akteur:innen selbst. Die Norm, Schule und Unterricht grundsätzlich inklusiv zu gestalten, wird heute in den meisten Schulgesetzen der Bundesrepublik formuliert. Zugleich finden sich dort weitere Normen, wie u. a. die der hierarchischen Unterscheidung der Schüler:innen entlang von Leistung. Mithin liegen bereits auf dieser formalen Ebene Widersprüche vor, die in der Praxis von den sozialen Akteur:innen gleichzeitig zu bearbeiten sind. Bezogen auf die Praxis lassen sich Inklusion und Partizipation - in Relation zu Exklusion und Behinderung - rekonstruieren. Dass diese Relation hochkomplex und zugleich ambivalent ist, zeigen Forschungsergebnisse, die mit dem Ansatz generiert wurden. So konnten unterrichtliche Differenzkonstruktionen von Lehrpersonen und Schüler:innen, also von Unterrichtsmilieus rekonstruiert werden, die an der Partizipation von Schüler:innen mit zugeschriebenem sonderpädagogischem Förderbedarf orientiert sind, die jedoch durch die enge (körperliche) Begleitung dieser Schüler:innen durch Sonderpädagog:innen zugleich mit einer Exklusion aus Peerinteraktionen einhergeht, die sich vielfach durch Distanzierungen von der Schüler:innenrolle auszeichnen. Diese sinngenetisch rekonstruierten Differenzkonstruktionen werden anschließend mit dem Ziel, soziogenetische Erklärungen zu generieren, mit Praxen verglichen, deren soziale und materiale sowie auch normative Kontexte sich unterscheiden, z. B. mit solchen aus Schulen, die dem formalen Anspruch nach exklusiv organisiert sind. Derartige Vergleiche eröffnen, wenn sie über Einzelfälle hinausgehen, Erklärungen für die Genese unterschiedlicher Differenzkonstruktionen und damit verbundener Ermöglichung und/oder Behinderung von Inklusion und Partizipation. Die rekonstruierten Praxen werden zudem mit den Normen und Erwartungen relationiert, die die sozialen Akteur:innen selbst für ihre Praxis haben. So zeigt Tanja Sturm (2021) z. B. auf der Grundlage einer Gruppendiskussion mit Schüler:innen einer Sekundarschule, dass diese die Norm teilen, dass inkorrekte Berechnungen von Schüler:innen im Mathematikunterricht keinen Anlass für klassenöffentliche Bloßstellungen sein sollen. In ihrer unterrichtlichen Praxis, dies konnte mittels Unterrichtsvideografien rekonstruiert werden, werden sie dieser Norm jedoch nicht gerecht; sie lachen über die Antwort einer Mitschülerin. Hier zeigt sich eine Diskrepanz zwischen ihrer Norm und ihrer Praxis. Interessant ist, dass die Mathematiklehrerin dieser Norm der Schüler:innen in ihrer Praxis gerecht wird.

In der praxeologisch-wissenssoziologischen Analyse bzw. den komparativen Vergleichen wird unterschieden, wer eine Norm formuliert, wie z. B. die Gesellschaft in Form kodifizierter Erwartungen und Regeln und/oder die Akteur:innen der Praxis. Von diesen beiden Akteur:innen sind die Wissenschaft bzw. die wissenschaftlichen Akteur:innen zu unterscheiden, die im Rahmen ihrer Forschungspraxis ebenfalls zu Bewertungen aufgefordert sind. Diese erfolgen dabei auf der Grundlage der skizzierten Vergleiche der unterschiedlichen Praxen sowie der Bearbeitung der Diskrepanz von Norm und Habitus in der Praxis. Die auf diesem Wege erkannten Gemeinsamkeiten und Unterschiede der Praxen bzw. der Orientierungsrahmen im weiteren Sinne vergleichbarer und/oder strukturell verschiedener Kontexte werden dann entlang 
$\mathrm{zu}$ explizierender theoretischer Perspektiven vergleichend bewertet (vgl. Bohnsack 2020, S. 110 ff.). Die praxeologisch-wissenssoziologisch generierten Ergebnisse ermöglichen eine (reflexive) Auseinandersetzung der Akteur:innen mit ihrer Praxis ebenso wie den Vergleich mit Praxen anderer, in strukturell vergleichbaren und/ oder unterschiedlichen Kontexten. Den Prämissen des Ansatzes folgend, führen derartige explizite Auseinandersetzungen mit Praxen nicht unmittelbar zu habituellen Veränderungen. Letztgenannte bedürfen auch einer Veränderung des institutionellgesellschaftlichen Rahmens bzw. seiner Normen. So zeigen Vergleiche mit unterrichtlichen Praxen eingliedriger Schulsysteme, auch für den Primarstufenbereich, dass Leistungsdifferenzen den Schüler:innen nicht vergleichbar individualisiert und hierarchisiert zugeschrieben werden, wie dies in den Unterrichtspraxen erfolgt, die in einem Schulsystem mit mehrgliedriger Grundstruktur hervorgebracht werden (vgl. Sturm 2019).

Die konstitutive Diskrepanz von Norm und Praxis der Praxeologischen Wissenssoziologie, auf deren Grundlage Inklusion und Partizipation als explizierte Normen einerseits und als habituell in der Praxis hervorgebracht andererseits unterschieden werden, unterscheidet sich gegenüber dem Verständnis der evidenzbasierten Bildungsforschung darin, dass die Wissenschaft keine Normen für die Praxis entwickelt, sondern die habituelle Bearbeitung (unterschiedlicher) Normen und Erfahrungen rekonstruiert werden. Mit den diskursanalytisch fundierten Dis_Ability Studies teilt die Praxeologische Wissenssoziologie die Perspektive, dass die Genese von Praxen nicht in der individuellen Verantwortung einzelner Subjekte liegt, sondern gesellschaftlich resp. kollektiv und zugleich kontextualisiert ist. Die Zugänge unterscheiden sich darin, dass Normen in Relation zu Abweichungen in den Dis_Ability Studies als Produkte der Praxis verstanden werden, während Normen in der Praxeologischen Wissenssoziologie v.a. explizit vorliegende Erwartungen - unterschiedlicher sozialer Akteur:innen - beschreiben, die in der Praxis v. a. handlungspraktisch bearbeitet werden.

\section{Wissenschaftliche Bewertung der Normen und Praxen schulisch- unterrichtlicher Inklusion und Partizipation}

Dieser knappe Einblick in ausgewählte erziehungswissenschaftliche Verständnisse der Relation von Norm und Praxis schulisch-unterrichtlicher Inklusion und Partizipation zeigt nicht nur, dass die hierauf aufbauenden Gegenstandsverständnisse different sind, sondern auch, dass die Genese von Normen und Praxen unterschiedlichen Akteur:innen zugeschrieben wird ebenso wie deren Bewertung. Letztgenanntes geht mit je eigenen Formen des Einbezugs der Perspektiven jener sozialer Akteur:innen einher, deren Praxis und/oder Normen von Inklusion und Partizipation Gegenstand der Forschung sind. Diese sollen abschließend verglichen werden.

In der evidenzbasierten Bildungsforschung wird das, was als Inklusion - bzw. Facette von Inklusion - und Partizipation in (Grund-)Schule und Unterricht verstanden wird, wesentlich in der Wissenschaft entwickelt, indem die Wirksamkeit theoretischer Ausarbeitungen statistisch ermittelt wird. Erklärungen für Diskrepanzen zwischen theoretisch und empirisch ermittelten Normen und Praxen werden zwar 
ursächlich bei den pädagogischen Akteur:innen verortet, die die Normen (eingeschränkt bzw. ungenügend) in der Praxis umsetzen. Nach möglichen Begründungen und/oder eigenen Erklärungen für die Diskrepanzen werden sie jedoch nicht befragt. Darin zeigt sich ein hierarchisches Verständnis von Wissenschaft und Praxis, in dem erstgenannte nicht nur die Konzepte für die Praxis bereitstellt, sondern diese auch entlang der von ihr generierten Maßstäbe bewertet. Die Perspektiven und die Erfahrungen der Praktiker:innen werden dabei allenfalls als Erklärungen für nicht gelingende Praxen herangezogen. Wissenschaftliches Wissen wird dem der Praxis damit übergeordnet bzw. als höherwertig verstanden.

Die Dis_Ability Studies verstehen Normen und Abweichungen hingegen komplementär und als der Praxis inhärente Diskursformationen, die die Partizipation von Subjekten behindern und/oder ermöglichen. Dies wird von einem deskriptivanalytisch arbeitenden gesellschaftskritischen Wissenschaftsverständnis angeleitet, das an dem Ziel orientiert ist, aufzuzeigen, welche Diskursformationen Normalität und Behinderungen hervorbringen. Diese zu überwinden wird v.a. als ein gesellschaftspolitischer und nicht als individueller Prozess verstanden. Die Wissenschaft verfügt dabei über die Zeit und die Methoden der differenzierten Analyse, die sie der Gesellschaft und der Praxis zur Verfügung stellt. Dieses Wissen hat insofern ,transformatorisches Potenzial“" (Hoffmann 2019, S. 100), als die Analyse Ausgangspunkt für gesellschaftliche, v. a. politische Veränderungen darstellen kann.

Die Praxeologische Wissenssoziologie, der die Annahme eines konstitutiven Spannungsverhältnisses von Norm und Praxis zugrunde liegt, geht davon aus, dass Normen und Praxen unterschiedlichen Logiken folgen, deren Diskrepanz von den sozialen Akteur:innen v. a. handlungspraktisch bearbeitet wird. Dieser kultursoziologische Zugang stellt v.a. einen begrifflichen Rahmen für menschliche Sozialität bereit, ohne, dass ihm ein vergleichbarer gesellschaftskritischer Bezug inhärent ist wie den Dis_Ability Studies. Hieran schließt das Verständnis und das Vorgehen der sozialwissenschaftlichen Bewertung sozialer Praxen an, die zunächst einen Vergleich der Normen, die die Akteur:innen selbst an ihre Praxen haben und die vonseiten der Organisation und/oder Institution an diese gestellt werden, vergleichend betrachtet. Eine sozialwissenschaftliche Bewertung fokussiert die Rekonstruktion unterschiedlicher Formen der Bearbeitung der Spannungsverhältnisse von Norm und Habitus und greift dabei die zuvor komparativ betrachteten Erkenntnisse auf, bevor sie diese mit dem zu explizierenden wissenschaftlichen Standort, von dem aus sie bewertet, vergleicht. Das wissenschaftlich generierte Wissen wird dabei nicht als höherwertiges verstanden, sondern v.a. als eines, das jenes Wissen, über das die sozialen Akteur:innen implizit verfügen, expliziert und so reflexiv zugänglich macht. Dieses (handlungsleitende) Wissen der unterrichtlichen Akteur:innen und seine Genese stellen dabei den zentralen Gegenstand dar (vgl. Bohnsack 2020, S. $110 \mathrm{ff}$.).

Dieser kurze einführende und vergleichende Einblick in erziehungswissenschaftliche Konzeptionen der Relation von Norm und Praxis von Inklusion und Partizipation verdeutlicht nicht nur, dass diese differente Gegenstandsverständnisse hervorbringen und unterschiedlichen Erkenntnisinteressen folgen, sondern auch, dass die Relation von Wissenschaft und Praxis und damit verbundene Formen der Bewertung different konzipiert werden. Letztgenanntes zeigt sich wesentlich darin, wie das Erfahrungswissen der (professionellen) Praktiker:innen berücksichtigt wird bzw. als konstituti- 
ves Element für Praxen - gegenüber dem Wissen der Forschung resp. der Wissenschaft - anerkannt wird. Die unterschiedlichen Zugänge und die damit verbundenen differenten Forschungspraxen sollen an dieser Stelle nicht bewertet werden. Vor dem Hintergrund des Vergleichs lässt sich jedoch der grundschulpädagogischen Theoriebildung und praktischen Forschung zu den Themen Inklusion und Partizipation empfehlen, die von ihnen jeweils zugrunde gelegten (Wissenschafts-)Verständnisse von Normen und (Forschungs-)Praxen offen zu legen. So kann gegenüber Dritten, allen voran gegenüber den Beforschten, nachvollzieh- und diskutierbar gemacht und auch partizipativ entschieden werden, wie die Zusammenarbeit von Wissenschaft und Praxis in einem konkreten Forschungsprojekt von den Akteur:innen warum wie gestaltet wird. Dies stellt eine Möglichkeit dar, Inklusion und Partizipation nicht nur als Forschungsgegenstände der Praxen anderer zu betrachten, sondern als Prinzipien der Forschungspraxis.

Funding Open Access funding enabled and organized by Projekt DEAL.

Open Access Dieser Artikel wird unter der Creative Commons Namensnennung 4.0 International Lizenz veröffentlicht, welche die Nutzung, Vervielfältigung, Bearbeitung, Verbreitung und Wiedergabe in jeglichem Medium und Format erlaubt, sofern Sie den/die ursprünglichen Autor(en) und die Quelle ordnungsgemäß nennen, einen Link zur Creative Commons Lizenz beifügen und angeben, ob Änderungen vorgenommen wurden.

Die in diesem Artikel enthaltenen Bilder und sonstiges Drittmaterial unterliegen ebenfalls der genannten Creative Commons Lizenz, sofern sich aus der Abbildungslegende nichts anderes ergibt. Sofern das betreffende Material nicht unter der genannten Creative Commons Lizenz steht und die betreffende Handlung nicht nach gesetzlichen Vorschriften erlaubt ist, ist für die oben aufgeführten Weiterverwendungen des Materials die Einwilligung des jeweiligen Rechteinhabers einzuholen.

Weitere Details zur Lizenz entnehmen Sie bitte der Lizenzinformation auf http://creativecommons.org/ licenses/by/4.0/deed.de.

\section{Literatur}

Ainscow, M. (2008). Teaching for diversity. The next big challenge. In F. M. Connelly, F. M. He \& J. Phillion (Hrsg.), The sage handbook of curriculum and instruction (S. 240-258). Los Angeles, London, New Delhi, Singapore: SAGE.

Becker, U. (2015). Die Inklusionslüge. Bielefeld: transcript.

Bohnsack, R. (2017). Praxeologische Wissenssoziologie. Opladen, Toronto: Budrich.

Bohnsack, R. (2020). Professionalisierung in praxeologischer Perspektive: Zur Eigenlogik der Praxis in Lehramt, Sozialer Arbeit und Frühpädagogik. Opladen, Toronto: Budrich, utb.

Buchner, T., Pfahl, L., \& Traue, B. (2015). Zur Kritik der Fähigkeiten: Ableism als neue Forschungsperspektive der Disability Studies und ihrer Partner_innen. Inklusion Online,(2). https://www.inklusiononline.net/index.php/inklusion-online/article/view/273/256. Zugegriffen: 25. Sept. 2021.

Erevelles, N. (2005). Understanding curriculum as normalizing text: disability studies meet curriculum theory. Journal of Curriculum Studies, 37(4), 421-439.

Foucault, M. (1976). Überwachen und Strafen. Die Geburt des Gefängnisses. Frankfurt/Main: Suhrkamp. Foucault, Michel (2020). Archäologie des Wissens. Frankfurt/Main: Suhrkamp Taschenbuch Wissenschaft. Fritzsche, B. (2018). Inklusion als Anerkennung einer primären Verletzbarkeit. Zum Ertrag Judith Butlers Anerkennungskonzept für die Analyse von inkludierenden und exkludierenden Effekten pädagogischer Praktiken. In T. Sturm \& M. Wagner-Willi (Hrsg.), Handbuch schulische Inklusion (S. 61-76). Opladen, Berlin, Toronto: Budrich. 
Fritzsche, B., Köpfer, A., Wagner-Willi, M., Böhmer, A., Nitschmann, H., Lietzmann, C., \& Weitkämpfer, F. (Hrsg.). (2021). Inklusionsforschung zwischen Normativität und Empirie. Abgrenzungen und Brückenschläge. Opladen, Berlin, Toronto: Budrich.

Gräsel, C. (2011). Was ist Empirische Bildungsforschung? In H. Reinders, H. Ditton, C. Gräsel \& B. Gniewiosz (Hrsg.), Empirische Bildungsforschung. Strukturen und Methoden (S. 13-27). Wiesbaden: Springer.

Grosche, M. (2015). Was ist Inklusion? Ein Diskussions- und Positionsartikel zur Definition von Inklusion aus Sicht der empirischen Bildungsforschung. In P. Kuhl, P. Stanat, B. Lütje-Klose, C. Gresch, H. A. Pant \& M. Prenzel (Hrsg.), Inklusion von Schülerinnen und Schülern mit sonderpädagogischem Förderbedarf in Schulleistungserhebungen (S. 17-39). Wiesbaden: Springer.

Hartmann, U., Decristan, J., \& Klieme, E. (2016). Unterricht als Feld evidenzbasierter Bildungspraxis? Herausforderungen und Potenziale für einen wechselseitigen Austausch von Wissenschaft und Schulpraxis. Zeitschrift für Erziehungswissenschaft, 19(1), 179-199.

Hinz, A., \& Boban, I. H. (2003). Index für Inklusion. Lernen und Teilhabe in der Schule der Vielfalt entwickeln. http://www.eenet.org.uk/resources/docs/Index\%20German.pdf. Zugegriffen: 25. Sept. 2021.

Hirschberg, M. und Köbsell, S. (2021). Disability Studies in Education: Normalität/en im inklusiven Unterricht und im Bildungsbereich hinterfragen. In: Köpfer, A., Powell, J. J. W. und Zahnd, R. (Hrsg.), Handbuch Inklusion international: Globale, nationale und lokale Perspektiven auf Inklusive Bildung: Globale, nationale und lokale Perspektiven auf Inklusive Bildung Handbook Inclusion International: Global, National and Local Perspectives on Inclusive Education. Opladen, Berlin, Toronto: Budrich. S. 127-146.

Hoffmann, T. (2019). Inklusive Schule, exklusive Gesellschaft? Soziologische Lesarten von Inklusion und Exklusion. In K. Müller \& S. Gingelmaier (Hrsg.), Kontroverse Inklusion. Ansprüche, Umsetzungen und Widersprüche in der Schulpädagogik (S. 54-77). Weinheim, Basel: Beltz Juventa.

Huber, C., \& Grosche, M. (2012). Das response-to-intervention-Modell als Grundlage für einen inklusiven Paradigmawechsel in der Sonderpädagogik. Zeitschrift für Heilpädagogik, 8, 312-322.

Katzenbach, D. (2015). Zu den Theoriefundamenten der Inklusion - Eine Einladung zum Diskurs aus der Perspektive der kritischen Theorie. In I. Schnell (Hrsg.), Herausforderung Inklusion. Theoriebildung und Praxis (S. 19-32). Bad Heilbrunn: Klinkhardt.

Köbsell, S. (2012). Integration/Inklusion aus Sicht der Disability Studies: Aspekte aus der internationalen und der deutschen Diskussion. In K. Rathgeb (Hrsg.), Disability Studies (S. 39-54). Wiesbaden: Springer.

Köbsell, S. (2015). Disability Studies in Education. Zeitschrift für Inklusion, (2). https://www.inklusiononline.net/index.php/inklusion-online/article/view/275/258. Zugegriffen: 25. Sept. 2021.

Koller, H.-C. (2017). Grundbegriffe, Theorien und Methoden der Erziehungswissenschaft. Eine Einführung. Stuttgart: Kohlhammer.

Linnemann, T., Mecheril, P., \& Nikolenko, A. (2013). Rassismuskritik. Begriffliche Grundlagen und Handlungsperspektiven in der politischen Bildung. Zeitschrift für internationale Bildungsforschung und Entwicklungspädagogik, 36(2), 10-14.

Luhmann, N., \& Schorr, K.E. (1982). Das Technologiedefizit der Erziehung und die Pädagogik. In N. Luhmann \& K. E. Schorr (Hrsg.), Zwischen Technologie und Selbstreferenz. Fragen an die Pädagogik (S. 11-40). Frankfurt/Main: Suhrkamp.

Mannheim, K. (1980). Strukturen des Denkens. Frankfurt/Main: Suhrkamp.

Meseth, W., Casale, R., Tervooren, A., \& Zirfas, J. (Hrsg.). (2019). Normativität in der Erziehungswissenschaft. Wiesbaden: Springer.

Müller, K., \& Pfrang, A. (2021). Risiken und Nebenwirkungen einer naiv evidenzbasierten Grundschulpädagogik zu Inklusion und Partizipation. Zeitschrift für Grundschulforschung, 14, 407-420. https:// doi.org/10.1007/s42278-021-00118-7.

Ricken, N. (2018). Konstruktionen der ,Leistung‘. Zur (Subjektivierungs-)Logik eines Konzepts. In S. Reh \& N. Ricken (Hrsg.), Leistung als Paradigma. Zur Entstehung und Transformation eines pädagogischen Konzepts (S. 43-60). Wiesbaden: Springer.

Sturm, T. (2019). Constructing and addressing differences in inclusive schooling-comparing cases from Germany, Norway and the United States. International Journal of Inclusive Education, 23(6), $656-669$.

Sturm, T. (2015). Rekonstruktiv-praxeologische Schul- und Unterrichtsforschung im Kontext von Inklusion. In: Inklusion Online, 4/2015. https://www.inklusion-online.net/index.php/inklusion-online/ article/view/321. Zugegriffen: 25. Sept. 2021. 
Sturm, T. (2021). Praxeologisch-wissenssoziologische Unterrichtsforschung: Norm und Habitus in fachunterrichtlichen Praxen der Sekundarstufe in Kanada. Zeitschrift für Qualitative Forschung, 22(2), 258-273.

Tervooren, A. (2000). Differenz anders gesehen: Studien zu Behinderung. Vierteljahreszeitschrift für Heilpädagogik und ihre Nachbargebiete, 69(3), 316-319.

Thiel, F., Cortina, K. S., \& Pant, H. A. (2014). Steuerung im Bildungssystem im internationalen Vergleich. In R. Fatke \& J. Oelkers (Hrsg.), Das Selbstverständnis der Erziehungswissenschaft: Geschichte und Gegenwart (S. 123-138). Weinheim: Beltz Juventa.

Tillmann, K.-J. (2016). Empirische Bildungsforschung in der Kritik - ein Überblick über Themen und Kontroversen. Zeitschrift für Erziehungswissenschaft, 19(1), 5-22.

Tremain, S. (Hrsg.). (2008). Foucault an the government of disability. Ann Arbor: The University of Michigan Press.

Tröhler, D. (2019). Bildungsstandards oder die Neudefinition von Schule. Entstehung, Verbreitung und Folgen einer globalen bildungspolitischen Sprache. In J. Zuber, H. Altrichter \& M. Heinrich (Hrsg.), Bildungsstandards zwischen Politik und schulischem Alltag (S. 3-24). Wiesbaden: Springer.

UN (2008). Übereinkommen über die Rechte von Menschen mit Behinderungen. (dreisprachige Fassung im Bundesgesetzblatt Teil II Nr. 35 vom 31.12.2008). (Manuskriptdruck). http://www.un.org/depts/ german/uebereinkommen/ar61106-dbgbl.pdf. Zugegriffen: 25. Sept. 2021.

Voß, S., Blumenthal, Y., Sikora, S., Mahlau, K., Diehl, K., \& Hartke, B. (2014). Rügener Inklusionsmodell (RIM) - Effekte eines Beschulungsansatzes nach dem Respone to Intervention-Ansatz auf die Rechen- und Leseleistungen von Grundschulkindern. Empirische Sonderpädagogik, 6(2), 114-132.

Vossen, A., \& Krizan, A. (2021). Response-to-Intervention als Rahmenmodell schulsicher Lernförderung. In J. Kuhl, A. Vossen, N. Hartung \& C. Wittich (Hrsg.), Evidenzbasierte Förderung bei Lernschwierigkeiten in der Grundschule (S. 18-27). München: Reinhardt.

Waldschmidt, A. (2005). Disability Studies: individuelles, soziales und/oder kulturelles Modell von Behinderung? Psychologie und Gesellschaftskritik, 29(1), 9-31.

Waldschmidt, A. (2007). Macht - Wissen - Körper. Anschlüsse an Michel Foucault in den Disability Studies. In A. Waldschmidt \& W. Schneider (Hrsg.), Disability Studies, Kultursoziologie und Soziologie der Behinderung. Erkundungen in einem neuen Forschungsfeld (S. 55-77). Bielefeld: transcript.

Waldschmidt, A. (2012). Normalität - Macht - Barrierefreiheit. Zur Ambivalenz der Normalisierung. In A. Tervooren \& J. Weber (Hrsg.), Wege zur Kultur. Barrieren und Barrierefreiheit in Kultur-und Bildungseinrichtungen (S. 52-66). Köln, Weimar, Wien: Böhlau.

Waldschmidt, A. (2020). Disability Studies. In C. Kammler, R. Parr \& J. U. Scheider (Hrsg.), Foucault Handbuch. Leben - Werk - Wirkung (S. 457-462). Berlin: J.B. Metzler.

Wansing, G. (2007). Behinderung: Inklusions- oder Exklusionsfolge? Zur Konstruktion paradoxer Lebensläufe in der modernen Gesellschaft. In A. Waldschmidt \& W. Schneider (Hrsg.), Disability Studies, Kultursoziologie und Soziologie der Behinderung. Erkundungen in einem neuen Forschungsfeld (S. 275-296). Bielefeld: transcript.

Weyers, S. (2019). Menschenrechte als normative Leitkategorie der Pädagogik. In W. Meseth, R. Casale, A. Tervooren \& J. Zirfas (Hrsg.), Normativität in der Erziehungswissenschaft (S. 71-93). Wiesbaden: Springer.

Wittich, C., \& Kuhl, J. (2021). Grundlagen der evidenzbasierten Förerung bei Lernschwierigkeiten in der inklusiven Schulpraxis. In J. Kuhl, A. Vossen, N. Hartung \& C. Wittich (Hrsg.), Evidenzbasierte Förderung bei Lernschwierigkeiten in der Grundschule (S. 7-17). München: Reinhardt.

Wrana, D. (2019). Die Normativität der Inklusion - ein Essay. Inklusion Online, 10(2). https://www. inklusion-online.net/index.php/inklusion-online/article/view/532. Zugegriffen: 23. Aug. 2021. 